\title{
Representation of Noncommutative Phase Space
}

\author{
Kang $\mathrm{Li}^{a 1}$, Jianhua Wang ${ }^{b}$ and Chiyi Chen ${ }^{c}$ \\ ${ }^{a}$ Department of Physics, Hangzhou Teacher's College, Hangzhou, 310036, P.R. China \\ ${ }^{b}$ Shaanxi College of Science and Engineer, Hanzhong, 723000,P.R. China \\ c Shanghai Astronomical Observatory, Chinese Academy of Sciences, Shanghai 200030, PR \\ China
}

\begin{abstract}
The representations of the algebra of coordinates and momenta of noncommutative phase space are given. We study, as an example, the harmonic oscillator in noncommutative space of any dimension. Finally the map of Schrödinger equation from noncommutative space to commutative space is obtained.
\end{abstract}

PACS number: $03.65 \mathrm{Bz}, 11.90 .+\mathrm{t}$

\footnotetext{
${ }^{1}$ kangli@hztc.edu.cn
} 
Recently, there has been much interest in the study of physics in noncommutative space (NCS) [1], not only because the NCS is necessary when one studies the low energy effective theory of D-brane with B field background, but also because in the very tiny string scale or at very high energy situation, the effects of non commutativity of space may appear. In literature the noncommutative quantum mechanics and noncommutative quantum field theory have been studied extensively, and the main approach is based on the Weyl-Moyal correspondence which amounts to replacing the usual product by a star product in non-commutative space.

In this paper, rather than studying the star product, we analyze the noncommutative effects in the usual quantum mechanics phase space. The usual method to do this is to use the Seiberg-Witten (SW) type map[1], that is to map the Gauge field or energy momentum tensor to their counter-part in commutative space. This method has been developed a lot in the past few years [8]. Our aim in this paper is to give a convenient method, albeit equivalent to the SW map, to study the noncommutative effects using commutative space coordinates. First we will give a representation of the noncommutative coordinates and noncommutative momenta in terms of the commutative coordinates and momenta of usual quantum mechanics. Then, as an example, we study the noncommutative harmonic oscillator in any dimension. At last, we give the representation of the Hamiltonian operator of noncommutative space and, as a consequence, the Schrödinger equation containing the noncommutative effects is obtained.

In the usual commutative space (say n dimensional space), the coordinates and momenta in quantum mechanics have the following commutation relations:

$$
\left\{\begin{array}{l}
{\left[x_{i}, x_{j}\right]=0,} \\
{\left[p_{i}, p_{j}\right]=0,} \\
{\left[x_{i}, p_{j}\right]=i \hbar \delta_{i j} .}
\end{array}\right.
$$

At very tiny scales, say string scale, the space may not commute anymore. Let us denote the operators of coordinates and momenta in noncommutative space as $\hat{x}$ and $\hat{p}$ respectively, then the $\hat{x}_{i}$ and $\hat{p}_{i}$ will have the following algebra relations, if both space-space and momentum-momentum non-commutativities are considered.

$$
\left\{\begin{array}{l}
{\left[\hat{x}_{i}, \hat{x}_{j}\right]=i \hbar \Theta_{i j},} \\
{\left[\hat{p}_{i}, \hat{p}_{j}\right]=i \hbar \bar{\Theta}_{i j},} \\
{\left[\hat{x}_{i}, \hat{p}_{j}\right]=i \hbar \delta_{i j} .}
\end{array}\right.
$$

where $\left\{\Theta_{i j}\right\}$ and $\left\{\bar{\Theta}_{i j}\right\}$ are totally antisymmetric matrices with very small elements representing the noncommutative property of the space and momentum in noncommutative 
phase space. Using the equation (11) and (2), we can study the representations of the noncommutative $\hat{x}$ and $\hat{p}$ in term of $x$ and $p$. Once these representations are obtained, then the noncommutative problems can be changed into problems in the usual commutative space which we are familiar with. In order to do so, let us give an ansatz as follows (the summation between repeated indices is implied in this paper ).

$$
\left\{\begin{array}{c}
\hat{x}_{i}=a_{i j} x_{j}+b_{i j} p_{j}, \\
\hat{p}_{i}=c_{i j} x_{j}+d_{i j} p_{j} . \\
\\
i, j=1,2 \ldots n
\end{array}\right.
$$

In matrix form, we have

$$
\left(\begin{array}{l}
\hat{x} \\
\hat{p}
\end{array}\right)=\left(\begin{array}{ll}
A & B \\
C & D
\end{array}\right)\left(\begin{array}{l}
x \\
p
\end{array}\right)
$$

where $A=\left\{a_{i j}\right\}, B=\left\{b_{i j}\right\}, C=\left\{c_{i j}\right\}$ and $D=\left\{d_{i j}\right\}$ are $n \times n$ matrices.

Now let us calculate the exact form of the representation matrices. For this, we insert equation (3) into equation (21), and using equation (11) we obtain

$$
\begin{aligned}
& A B^{T}-B A^{T}=\Theta \\
& C D^{T}-D C^{T}=\bar{\Theta} \\
& A D^{T}-B C^{T}=\mathbf{1}
\end{aligned}
$$

where $\mathbf{1}$ is the identity matrix and the upper index $T$ of a matrix means its transpose. In the above relations, the matrices $A$ and $D$ could be chosen as proportional to identity (scaling factors), so we denote them by $\alpha$ and $\beta$ respectively ${ }^{2}$. Then the relations above change into,

$$
\begin{aligned}
& \alpha\left(B^{T}-B\right)=\Theta \\
& \beta\left(C-C^{T}\right)=\bar{\Theta} \\
& B C^{T}=(\alpha \beta-1) \mathbf{1}
\end{aligned}
$$

From first two equations of (6), we can see that if $B$ and $C$ are antisymmetric, then they will have explicit solutions, in fact if we assume $B$ and $C^{T}$ are commuting, then the third equation of the (6) is satisfied when $B$ and $C$ are either symmetric or antisymmetric. The case of $B$ and $C$ being both symmetric leads to the $\Theta=\bar{\Theta}=0$ which means our space is commutative. Thus, we choose $B$ and $C$ as antisymmetric matrices. Then we obtain

$$
\begin{aligned}
& B=-\frac{1}{2 \alpha} \Theta, \\
& C=\frac{1}{2 \beta} \bar{\Theta} .
\end{aligned}
$$

\footnotetext{
${ }^{2}$ Symmetry considerations tell us that these two constant matrices should be same. This will be shown to be the case in our generic discussion of noncommutative harmonic oscillator in n-dimensions in the later part of this paper
} 
Here the scaling constants $\alpha$ and $\beta$ should not be equal to zero. The last equation of (6) gives the relationship between $\bar{\Theta}$ and $\Theta$

$$
\Theta \bar{\Theta}=4 \alpha \beta(\alpha \beta-1) \cdot \mathbf{1}
$$

One can then put the general representation matrix of $(\hat{x}, \hat{p})$ in the commutative space as:

$$
\left(\begin{array}{cc}
\alpha \cdot \mathbf{1} & -\frac{1}{2 \alpha} \Theta, \\
\frac{1}{2 \beta} \bar{\Theta} & \beta \cdot \mathbf{1}
\end{array}\right)_{2 n \times 2 n}
$$

And equations (3) become,

$$
\left\{\begin{array}{c}
\hat{x}_{i}=\alpha x_{i}-\frac{1}{2 \alpha} \Theta_{i j} p_{j} \\
\hat{p}_{i}=\beta p_{i}+\frac{1}{2 \beta} \bar{\Theta}_{i j} x_{j} \\
i, j=1,2 \ldots n
\end{array}\right.
$$

When $\alpha=\beta=1$, it will correspond to $\bar{\Theta}=0$ ( refer to the next part of this paper), which is the case that is extensively studied in the literature where the space coordinates are non-commuting while momentum space is commuting.

The angular momentum of noncommutative space is given by,

$$
\hat{L}_{i}=\epsilon_{i j k} \hat{x}_{j} \hat{p}_{k}
$$

By using the representation equations (10), the $\hat{L}$ in usual commutative space can be written as,

$$
\begin{aligned}
\hat{L}_{i}= & \epsilon_{i j k}\left(\alpha \beta x_{j} p_{k}+\frac{\alpha}{2 \beta} x_{j} \bar{\Theta}_{k m} x_{m}\right. \\
& \left.-\frac{\alpha}{2 \beta} \Theta_{j l} p_{l} p_{k}-\frac{1}{4 \alpha \beta} \Theta_{j l} p_{l} \bar{\Theta}_{k m} x_{m}\right) .
\end{aligned}
$$

Or we can write it in vector form ,

$$
\begin{aligned}
\hat{\mathbf{L}}= & \alpha \beta \mathbf{x} \times \mathbf{p}-\frac{\alpha}{2 \beta}(\Theta \mathbf{p}) \times \mathbf{p} \\
& +\frac{\alpha}{2 \beta} \mathbf{x} \times(\bar{\Theta} \mathbf{x})-\frac{1}{4 \alpha \beta}(\Theta \mathbf{p}) \times(\bar{\Theta} \mathbf{x}) .
\end{aligned}
$$

And the commutation relations between $\hat{\mathbf{L}}$ and $\hat{\mathbf{x}}, \hat{\mathbf{p}}$ have the forms as follows,

$$
\begin{aligned}
{\left[\hat{L}_{i}, \hat{x}_{j}\right] } & =\left[\epsilon_{i k l} \hat{x}_{k} \hat{p}_{l}, \hat{x}_{j}\right] \\
& =i \hbar \epsilon_{i j k} \hat{x}_{k}+i \hbar \epsilon_{i k l} \Theta_{k j} \hat{p}_{l} \\
{\left[\hat{L}_{i}, \hat{p}_{j}\right] } & =\left[\epsilon_{i k l} \hat{x}_{k} \hat{p}_{l}, \hat{p}_{j}\right] \\
& =i \hbar \epsilon_{i j k} \hat{p}_{k}-i \hbar \epsilon_{i k l} \bar{\Theta}_{k j} \hat{x}_{l}
\end{aligned}
$$


Up to now, we have given the representation of coordinates and momenta of noncommutative space in terms of the coordinates and momenta in the usual quantum mechanics commutative space. Using these representations we can study the effects of non-commutativity in the usual space we are familiar with. As an illustrative example, let us study in detail the harmonic oscillator in noncommutative space with arbitrary dimensions $n$. In $n$ dimensional noncommutative space, the Hamiltonian of the harmonic oscillator has the form as,

$$
\hat{H}=\frac{1}{2 \mu} \hat{p}_{i} \hat{p}_{i}+\frac{1}{2} \mu \omega^{2} \hat{x}_{i} \hat{x}_{i}
$$

where the $\mu$ and $\omega$ represent the mass and angular frequency of the oscillator. Using the representation equations in (10), we obtain the expression of the $\hat{H}$ in the commutative space as,

$$
\begin{aligned}
\hat{H} & =\frac{\beta^{2}}{2 \mu} p_{i} p_{i}+\frac{\alpha^{2}}{2} \mu \omega^{2} x_{i} x_{i}+\left(\frac{1}{4} \mu \omega^{2} \Theta_{i j}+\frac{1}{4 \mu} \bar{\Theta}_{i j}\right)\left(p_{i} x_{j}+x_{j} p_{i}\right) \\
& +\frac{\mu \omega^{2}}{8 \alpha^{2}} \Theta_{i j} \Theta_{i l} p_{j} p_{l}+\frac{1}{8 \mu \beta^{2}} \bar{\Theta}_{i j} \bar{\Theta}_{i l} x_{j} x_{l}
\end{aligned}
$$

Again we can see that when $\alpha=\beta=1$, corresponding to $\bar{\Theta}_{i j}=0$, then the above Hamiltonian corresponds to the case where the space is non-commutative while the momenta are commuting. If we set further more $\Theta_{i j}=0$ then the result corresponds to the usual $n$ dimension harmonic oscillator in commutative space.

For convenience, the annihilation operators $a$ and creation $a^{\dagger}$ operators are introduced when one studies harmonic oscillator problem. Similarly, we would like to introduce their counterparts $\hat{a}$ and $\hat{a}^{\dagger}$ in noncommutative space and the relationship between them. The deformed annihilation-creation operators in $n$-dimensional noncommutative space are defined by

$$
\hat{a}_{i}=\sqrt{\frac{\mu \omega}{2}}\left(\hat{x}_{i}+\frac{i}{\mu \omega} \hat{p}_{i}\right) \quad \hat{a}_{i}^{\dagger}=\sqrt{\frac{\mu \omega}{2}}\left(\hat{x}_{i}-\frac{i}{\mu \omega} \hat{p}_{i}\right)
$$

It is easy to check that,

$$
\begin{aligned}
& {\left[\hat{a}_{i}, \hat{a}_{j}^{\dagger}\right]=\delta_{i j}+i \mu \omega \Theta_{i j}} \\
& {\left[\hat{a}_{i}, \hat{a}_{j}\right]=\left[\hat{a}_{i}^{\dagger}, \hat{a}_{j}^{\dagger}\right]=\frac{i}{2} \mu \omega\left(\Theta_{i j}-\frac{1}{\mu^{2} \omega^{2}} \bar{\Theta}_{i j}\right)}
\end{aligned}
$$

In order to keep Bose-Einstein statistics in noncommutative case we need $\hat{a}_{i}^{\dagger}$ and $\hat{a}_{j}^{\dagger}$ to be commuting, whence the consistency condition:

$$
\bar{\Theta}=\mu^{2} \omega^{2} \Theta
$$

Under this condition, the above commutators become

$$
\begin{aligned}
& {\left[\hat{a}_{i}, \hat{a}_{i}^{\dagger}\right]=1} \\
& {\left[\hat{a}_{i}, \hat{a}_{j}\right]=\left[\hat{a}_{i}^{\dagger}, \hat{a}_{j}^{\dagger}\right]=0 .}
\end{aligned}
$$


which are just the same as the ones in commutative space. But there is also an extra commutator

$$
\left[\hat{a}_{i}, \hat{a}_{j}^{\dagger}\right]=i \mu \omega \Theta_{i j} \text {, for } i \neq j .
$$

We point out here that this abnormal extra commutator is the reason of the existence of fractionally angular momentum [9], and it is consistent with all principles and Bose-Einstein statistics. Replacing (20) in (10) we have

$$
\left\{\begin{array}{c}
\hat{x}_{i}=\alpha x_{i}-\frac{1}{2 \alpha} \Theta_{i j} p_{j} \\
\hat{p}_{i}=\beta p_{i}+\frac{1}{2 \beta} \mu^{2} \omega^{2} \Theta_{i j} x_{j} . \\
i, j=1,2 \ldots n
\end{array}\right.
$$

As we know in the commutative space, the annihilation and creation operators can be expressed as

$$
a_{i}=\sqrt{\frac{\mu \omega}{2}}\left(x_{i}+\frac{i}{\mu \omega} p_{i}\right), \quad a_{i}^{\dagger}=\sqrt{\frac{\mu \omega}{2}}\left(x_{i}-\frac{i}{\mu \omega} p_{i}\right) .
$$

So from a straight calculation we get the relation between $\hat{a}_{i}, \hat{a}_{i}^{\dagger}$ and $a_{i}, a_{i}^{\dagger}$,

$$
\begin{aligned}
\hat{a}_{i}= & \frac{1}{2}(\alpha+\beta) a_{i}+\frac{i}{4} \mu \omega\left(\frac{1}{\beta}+\frac{1}{\alpha}\right) \Theta_{i j} a_{j} \\
& +\frac{1}{2}(\alpha-\beta) a_{i}^{\dagger}+\frac{i}{4} \mu \omega\left(\frac{1}{\beta}-\frac{1}{\alpha}\right) \Theta_{i j} a_{j}^{\dagger}, \\
\hat{a}_{i}^{\dagger}= & \frac{1}{2}(\alpha+\beta) a_{i}^{\dagger}-\frac{i}{4} \mu \omega\left(\frac{1}{\beta}+\frac{1}{\alpha}\right) \Theta_{i j} a_{j}^{\dagger} \\
& +\frac{1}{2}(\alpha-\beta) a_{i}-\frac{i}{4} \mu \omega\left(\frac{1}{\beta}-\frac{1}{\alpha}\right) \Theta_{i j} a_{j} .
\end{aligned}
$$

Causality considerations tell us that if a particle is annihilated in noncommutative space, and in order to view this phenomenon in commutative space, then it should correspond to some sort of annihilation in the latter space. That is to say $\hat{a}_{i}$ should only be some combination of $a_{i}$ 's. For the same reason, $\hat{a}_{i}^{\dagger}$ should also be some combination of $a_{i}^{\dagger}$ 's. So this forces us to set,

$$
\alpha=\beta=: \alpha .
$$

And then the equations in (25) become

$$
\begin{aligned}
& \hat{a}_{i}=\alpha a_{i}+\frac{i}{2 \alpha} \mu \omega \Theta_{i j} a_{j}, \\
& \hat{a}_{i}^{\dagger}=\alpha a_{i}^{\dagger}-\frac{i}{2 \alpha} \mu \omega \Theta_{i j} a_{j}^{\dagger} .
\end{aligned}
$$

Now we can discuss the Hamiltonian and angular momentum in term of annihilation and creation operators. It is easy to check that the Hamiltonian of harmonic oscillator in equation (16) has the form

$$
\begin{aligned}
\hat{H} & =\hbar \omega \hat{a}_{i}^{\dagger} \hat{a}_{i}+\frac{n}{2} \hbar \omega \\
& =\hbar \omega\left[\alpha^{2} a_{i}^{\dagger} a_{i}+i \mu \omega \Theta_{i j} a_{i}^{\dagger} a_{j}+\frac{1}{4 \alpha^{2}} \mu^{2} \omega^{2} \Theta_{i j} \Theta_{i l} a_{j}^{\dagger} a_{l}+\frac{n}{2}\right] .
\end{aligned}
$$


We can see that the vacuum energy (the last term) exists also in the noncommutative case, while the non-commutative effects appear in the second and third terms related to the $\Theta$. The angular momentum can be written as

$$
\begin{aligned}
\hat{L}_{i} & =\epsilon_{i j k} \hat{x}_{j} \hat{p}_{k}=\frac{i \hbar}{2} \epsilon_{i j k}\left(\hat{a}_{a} \hat{a}_{k}^{\dagger}+\hat{a}_{k}^{\dagger} \hat{a}_{j}\right) \\
& =-i \hbar \epsilon_{i j k} \hat{a}_{j}^{\dagger} \hat{a}_{k}-\frac{\hbar}{2} \mu \omega \epsilon_{i j k} \Theta_{j k} .
\end{aligned}
$$

The first term is identical in form to the commutative case. However, it includes noncommutative effects in the expressions of $\hat{a}_{k}$ and $\hat{a}_{j}^{\dagger}$ :

$$
-i \hbar \epsilon_{i j k} \hat{a}_{j}^{\dagger} \hat{a}_{k}=-i \hbar \epsilon_{i j k}\left\{\alpha^{2} a_{j}^{\dagger} a_{k}+\frac{i}{2} \mu \omega\left(\Theta_{k l} a_{j}^{\dagger} a_{l}-\Theta_{j l} a_{l}^{\dagger} a_{k}\right)+\frac{\mu^{2} \omega^{2}}{4 \alpha^{2}} \Theta_{j l} \Theta_{k m} a_{l}^{\dagger} a_{k}\right\} .
$$

The more important thing is the last term in equation (29). This is a new effect proper to noncommutative space. It tells us that in noncommutative space, the angular momentum has a non-zero "zero-point" angular momentum. For a given noncommutative space $(\Theta$ fixed), this "zero-point" angular momentum depends on the mass and angular frequency, so it can have a fractional value.

In fact, we should point out that the noncommutative effects always depend on the coordinates commutator matrix $\Theta$, and the matrix elements depend on the scaling constant $\alpha$. From (8), (201) and (26) we have

$$
\Theta_{i l} \Theta_{l j}=-\theta^{2} \delta_{i j}
$$

where

$$
\theta=\frac{2 \alpha}{\mu \omega} \sqrt{\left(1-\alpha^{2}\right)}
$$

and $\alpha$ should be less or equal to 1 .

When $n=2$, we obtain,

$$
\Theta=\left(\begin{array}{cc}
0 & \theta \\
-\theta & 0
\end{array}\right)
$$

Our results here for 2 dimensional space coincide with the results in reference $[9]$, if we make the following correspondence,

$$
\alpha \Leftrightarrow \xi^{-1}=\left(1+d d^{\prime} / 4\right)^{-\frac{1}{2}}
$$

and

$$
\begin{aligned}
& \Theta_{i j} \Leftrightarrow \xi^{-2} \Lambda_{N C}^{-2} d \epsilon_{i j}, \\
& \bar{\Theta}_{i j} \Leftrightarrow \xi^{-2} \Lambda_{N C}^{2} d^{\prime} \epsilon_{i j} .
\end{aligned}
$$

Where $\Lambda_{N C}$ is the noncommutative energy scale. When $d^{\prime}=0$, led to $\alpha=1$ and also $\bar{\Theta}=0$. 
When $n=3$, equation (30) has three solutions, which read,

$$
\left.\left.i): \Theta=\left(\begin{array}{ccc}
0 & \theta & 0 \\
-\theta & 0 & 0 \\
0 & 0 & 0
\end{array}\right), i i\right): \Theta=\left(\begin{array}{ccc}
0 & 0 & \theta \\
0 & 0 & 0 \\
-\theta & 0 & 0
\end{array}\right), i i i\right): \Theta=\left(\begin{array}{ccc}
0 & 0 & 0 \\
0 & 0 & \theta \\
0 & -\theta & 0
\end{array}\right)
$$

These solutions tell us that in our case the noncommutative 3 dimensional space for harmonic oscillators is reducible and, in fact, it is a direct sum of a 1-dimensional space and a 2-dimensional noncommutative space where the two subspaces are commutating.

When $n=4$, the equation (30) has six solutions which can be classified into three classes. The first class includes two solutions which have no free parameters. The two solutions for matrix $\Theta$ are give by

$$
\left(\begin{array}{cccc}
0 & 0 & 0 & \theta \\
0 & 0 & \theta & 0 \\
0 & -\theta & 0 & 0 \\
-\theta & 0 & 0 & 0
\end{array}\right),\left(\begin{array}{cccc}
0 & 0 & 0 & \theta \\
0 & 0 & -\theta & 0 \\
0 & \theta & 0 & 0 \\
-\theta & 0 & 0 & 0
\end{array}\right)
$$

These solutions correspond to a reducible space which is the direct sum of two 2-dimensional independent noncommutative spaces. The second class has two solutions which contain one free parameter and have the form

$$
\left(\begin{array}{cccc}
0 & \theta_{1} & \theta_{2} & 0 \\
-\theta_{1} & 0 & 0 & \theta_{2} \\
-\theta_{2} & 0 & 0 & \theta_{1} \\
0 & -\theta_{2} & -\theta_{1} & 0
\end{array}\right), \quad\left(\begin{array}{cccc}
0 & \theta_{1} & \theta_{2} & 0 \\
-\theta_{1} & 0 & 0 & \theta_{2} \\
-\theta_{2} & 0 & 0 & -\theta_{1} \\
0 & -\theta_{2} & \theta_{1} & 0
\end{array}\right)
$$

with $\theta_{1}$ and $\theta_{2}$ satisfy

$$
\theta_{1}^{2}+\theta_{2}^{2}=\theta^{2}
$$

The solutions of third class have two free parameters

$$
\left(\begin{array}{cccc}
0 & \vartheta_{1} & \vartheta_{2} & \vartheta_{3} \\
-\vartheta_{1} & 0 & \vartheta_{3} & -\vartheta_{2} \\
-\vartheta_{2} & -\vartheta_{3} & 0 & \vartheta_{1} \\
-\vartheta_{3} & \vartheta_{2} & -\vartheta_{1} & 0
\end{array}\right), \quad\left(\begin{array}{cccc}
0 & \vartheta_{1} & \vartheta_{2} & \vartheta_{3} \\
-\vartheta_{1} & 0 & -\vartheta_{3} & \vartheta_{2} \\
-\vartheta_{2} & \vartheta_{3} & 0 & -\vartheta_{1} \\
-\vartheta_{3} & -\vartheta_{2} & \vartheta_{1} & 0
\end{array}\right)
$$

with

$$
\vartheta_{1}^{2}+\vartheta_{2}^{2}+\vartheta_{3}^{2}=\theta^{2}
$$

The noncommutative spaces for these two class solutions are irreducible (at least they are non completely reducible for generic values of the free parameters).

In the last part of this paper, we would like to use the representation (10) to find a possible Schrödinger equation in commutative space which is equivalent to the the Schrödinger 
equation in noncommutative space. To begin with, let us discuss the Hamiltonian in noncommutative space which should have the following form

$$
\hat{H}=\frac{1}{2 \mu} \hat{p}_{i} \hat{p}_{i}+V\left(\hat{x}_{1}, \ldots \hat{x}_{n}\right)
$$

Up to the first order in $\Theta$ and $\bar{\Theta}$, we can write the noncommutative hamiltonian in terms of commutative variables as,

$$
\begin{aligned}
\hat{H}= & \frac{\alpha^{2}}{2 \mu} p_{i} p_{i}+V\left(\alpha x_{1}, \alpha x_{2} \ldots \alpha x_{n}\right) \\
& -\frac{1}{2 \mu} \bar{\Theta}_{i j} x_{i} p_{j}-\frac{1}{2 \alpha} \frac{\partial V\left(x_{1}, x_{2} \ldots x_{n}\right)}{\partial x_{i}} \Theta_{i j} p_{j} .
\end{aligned}
$$

In three dimensional space, the Hamiltonian [40] gives the Schrödinger equation as,

$$
i \hbar \frac{\partial \Psi(\mathbf{x}, t)}{\partial t}=\left\{-\frac{\alpha^{2} \hbar^{2}}{2 \mu} \nabla^{2}+V(\alpha \mathbf{x})-\frac{i \hbar}{2 \mu} \tilde{\mathbf{x}} \cdot \nabla+\frac{i \hbar}{2 \alpha} \nabla V \cdot \tilde{\nabla}\right\} \Psi(\mathbf{x}, t)
$$

where $\tilde{\mathbf{x}}_{i}=\bar{\Theta}_{i j} x_{j} \quad \tilde{\nabla}=\Theta_{i j} \partial_{j}$. When $\alpha=1$, which means $\bar{\Theta}=0$, then the transformation relations from noncommutative space to commutative space become,

$$
\begin{aligned}
& \hat{p}_{i}=p_{i} \\
& \hat{x}_{i}=x_{i}-\frac{1}{2} \Theta_{i j} p_{j}
\end{aligned}
$$

Thus, our result reduces to the situation discussed in many papers [10 13], where the Schrödinger equation reads,

$$
i \hbar \frac{\partial \Psi(\mathbf{x}, t)}{\partial t}=\left[\frac{p^{2}}{2 \mu}+V\left(\mathbf{x}-\frac{1}{2} \tilde{\mathbf{p}}\right)\right] \Psi(\mathbf{x}, t)
$$

with $\tilde{\mathbf{p}}_{i}=\Theta_{i j} p_{j}$.

In summary, we have achieved the following results in this paper. First, we got a general representation of any dimensional noncommutative phase space in term of the usual phase space in quantum mechanics. Then, as a special case, the noncommutative harmonic oscillator in any dimensions is studied in details, and we got an constraint for the matrix $\Theta$ related to the non-commutativity of the space. The results of a few low dimensional cases are given. At last, we discussed the form of Schrödinger equation which contains the noncommutative effects of the phase space. It should be pointed out that most of our results are not Lorentz invariant, because the time and the noncommutative spacial coordinates are assumed to commute in this paper. Nonetheless, the general relation, say equations (10) in the first part of this paper, can be used in the case of noncommutative space-time, where we can set $\hat{x}_{1}=i c \hat{t}$. 
Acknowledgements. This paper was written during Kang Li's visit to High Energy section of Abdus Salam ICTP, Trieste, Italy and to the Physics Department of La Plata National University, La Plata, Argentina, He would like to thank Prof. S.Randjbar-Daemi and Prof. Carlos Naon for scientific discussions. This work was supported partially by the National Nature Science Foundation of China (90303003) and the Nature Science Foundation of Zhejiang Provence, China (M103042;102011;102028). The author also recognizes the support of the Consejo Nacional de Investigaciones Científicas y Técnicas (CONICET), Argentina and the support of the Third World Academy of Sciences (TWAS).

\section{References}

[1] N. Seiberg and E. Witten, JHEP 9909:032 (1999) hep-th/9908142

[2] A. Connes, M. R. Douglas, A. Schwarz, JHEP 9802, 003 (1998) hep-th/9711162.

[3] M. R. Douglas, C. M. Hull, JHEP 9802, 008 (1998) hep-th/9711165

[4] F. Ardalan, H. Arfaei, M. M. Sheikh-Jabbari, JHEP 9902, 016 (1999) hep-th/9810072.

[5] S-C. Chu, P-M. Ho, Nucl. Phys. B550, 151 (1999) hep-th/9812219.

[6] S-C. Chu, P-M. Ho, Nucl. Phys. B568, 447 (2000) hep-th/9906192.

[7] V. Schomerus, JHEP 9906, 030 (1999) hep-th/9903205.

[8] R.Banerjee, C.Lee and H.S.Yang, Seiberg-witten-type Maps for currents and EnergyMomentum Tensors in Noncommuative Gauge Theories. hep-th/0312103

[9] Jian-zu Zhang, Phys. Lett. B584, 204(2004).

[10] M. Chaichian, M. M. Sheikh-Jabbari, A. Tureanu, Phys. Rev. Lett. 86, 2716 (2001) hep-th/0010175.

[11] J. Gamboa, M. Loewe, J. C. Rojas, Phys. Rev. D64, 067901 (2001) hep-th/0010220.

[12] J. Gamboa, M. Loewe, F. Mendez, J. C. Rojas, Int. J. Mod. Phys. A17, 2555 (1999) hep-th/0106125.

[13] B. Muthukumar, P. Mitra, Phys. Rev. D66, 027701 (2002) hep-ph/0204149.

[14] V. P. Nair, A. P. Polychronakos, Phys. Lett. B505, 267 (2001) hep-th/0011172. 
[15] D. Kochan, M. Demetrian, hep-th/0102050

[16] B. Morariu, A. P. Polychronakos, Nucl. Phys. B610, 531 (2001) hep-th/0102157.

[17] A. Hatzinikitas, I. Smyrnakis, J. Math. Phys. 43113 (2002) hep-th/0103074.

[18] S. Bellucci, A. Neressian, C. Sochichiu, Phys. Lett. B522, 345 (2001) hep-th/0106138.

[19] A. Smailagic, E. Spallucci, Phys. Rev. D65, 107701 (2002) hep-th/0108216.

[20] H. R. Christiansen, F. A. Schaposnik, Phys. Rev. D65, 086005 (2002) hep-th/0106181.

[21] C. Acatrinei, JHEP 0109, 007 (2001) hep-th/0107078.

[22] P-M. Ho, H-C. Kao, Phys. Rev. Lett. 88, 151602 (2002) hep-th/0110191.

[23] L. Alvarez-Gaume, S. R. Wadia, Phys. Lett. B501, 319 (2001) hep-th/0006219.

[24] A. Micu, M. M. Sheikh-Jabbari, JHEP 0101: 025 (2001) hep-th/0008057.

[25] I. F. Riad, M. M. Sheikh-Jabbari, JHEP 0008: 045 (2000) hep-th/0008132

[26] M. R. Douglas, N. A. Nekrasov, Rev. Mod. Phys. 73, 977 (2001) hep-th/0106048 